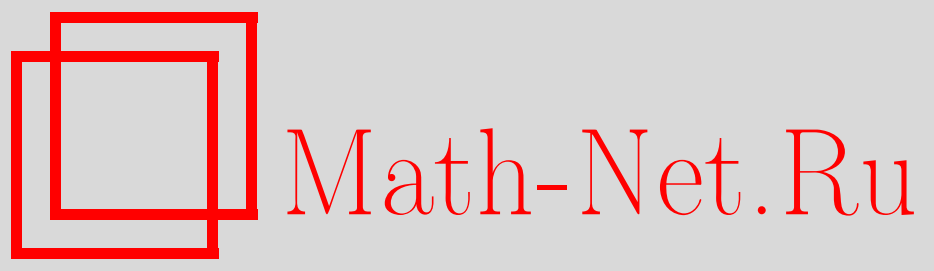

А. Х. Хачатрян, А. А. Хачатрян, Некоторые вопросы разрешимости уравнения Больцмана в рамках модели Шахова, ТМФ, 2017, том 191, номер 3, 441-455

DOI: https://doi.org/10.4213/tmf9243

Использование Общероссийского математического портала Math-Net.Ru подразумевает, что вы прочитали и согласны с пользовательским соглашением http://www.mathnet.ru/rus/agreement

Параметры загрузки:

IP : 35.173 .219 .149

26 апреля 2023 г., 17:07:02

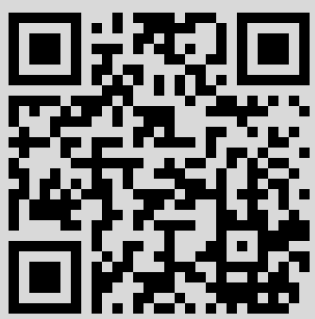




\title{
ФИЗИКА
}

Том 191, № 3

июнь, 2017

\section{НЕКОТОРЫЕ ВОПРОСЫ РАЗРЕШИМОСТИ УРАВНЕНИЯ БОЛЬЦМАНА В РАМКАХ МОДЕЛИ ШАХОВА}

\begin{abstract}
Рассматривается нелинейное уравнение Больцмана в рамках модели Шахова для классической задачи о течении газа в плоском слое. Задача сводится к системе нелинейных интегральных уравнений. Нелинейность рассматриваемой системы удается отчасти упростить путем перехода к новому аргументу, который зависит от решения самой задачи. Доказаны теорема существования единственного решения для линейной системы и теорема существования положительного решения для нелинейного уравнения Урысона. Найдены температурные скачки на нижних и верхних стенках в линейном и нелинейном случаях, которые, как оказалось, мало различаются.
\end{abstract}

Ключевые слова: нелинейность, монотонность, модельное уравнение, итерация, температурный скачок, кинетическая толщина.

DOI: https://doi.org/10.4213/tmf9243

\section{1. ВВЕДЕНИЕ}

Изучение вопросов разрешимости кинетического уравнения Больцмана имеет большое теоретическое и прикладное значение. Однако сложная структура истинного интеграла столкновений существенно усложняет изучение и решение уравнения Больцмана. Поэтому при рассмотрении ряда задач кинетической теории газов часто истинный интеграл столкновений заменяют приближенной моделью. Существует ряд кинетических моделей уравнения Больцмана, среди которых особое место занимают модель Бхатнагара-Гросса-Крука (БГК), эллипсоидально-статистическая модель (ЭС), модель Шахова, новая модифицированная модель Лиу (см. [1]-[8]). Каждая из моделей имеет свои преимущества и недостатки (см. [9]). В настоящее время наиболее широко используемой и популярной остается модель Шахова, поскольку она обобщает модель БГК.

Работа выполнена при финансовой поддержке ГКН МОН РА в рамках научного проекта № SCS 15T-1A033.

* Кафедра высшей математики и теоретической механики, Национальный аграрный университет Армении, Ереван, Армения. E-mail: aghavard59@mail.ru, hmayakk@bk.ru 
В настоящей работе рассматривается уравнение Больцмана в рамках модели Шахова для классической задачи о течении газа в плоском слое, ограниченном двумя параллельными пластинками. Задача сводится к системе нелинейных интегральных уравнений относительно четырех макропараметров - плотности, потока вектора энергии, температуры и скорости газа. Структура полученной системы достаточно сложна: с одной стороны, имеет место нелинейность интегральных уравнений системы, с другой - отсутствует свойство монотонности соответствующего интегрального оператора, что весьма усложняет построение неподвижной точки. Тем не менее мы предлагаем эффективный метод построения решения указанной нелинейной системы. Сначала удается отчасти упростить нелинейность рассматриваемой системы путем перехода к новому аргументу, который зависит от решения самой задачи. Описана схема возврата к исходному аргументу и определения кинетической толщины слоя.

Далее предлагается приближенное решение новой нелинейной системы. В предположении постоянства температуры задача сводится к системе линейных интегральных уравнений относительно плотности и вектора потока энергии газа. Доказана теорема о существовании и единственности решения полученной системы линейных интегральных уравнений со знакопеременным ядром. Затем в предположении постоянства вектора потока энергии и при заданном виде плотности задача сводится к нелинейному интегральному уравнению типа Урысона относительно функции, определяющей температуру газа. Доказано существование положительного решения этого уравнения. В конце статьи рассматривается линейная система интегральных уравнений, получаемая в результате линеаризации исходной нелинейной системы. Найдено максимальное значение расстояния между пластинками, при котором указанная система однозначно разрешима. Выполнены численные расчеты и найдены температурные скачки на стенках как в линейном, так и в нелинейном случае.

\section{2. ПОСТАНОВКА ЗАДАЧИ}

Рассмотрим задачу о течении газа в плоском слое, ограниченном твердыми параллельными пластинками $x=0$ и $x=d$. Обозначим через $f(x, \vec{s})$ искомую функцию распределения частиц по скоростям $\vec{s}=\left(s_{1}, s_{2}, s_{3}\right)$. В силу предполагаемой плоской геометрии функция распределения частиц $f$ не зависит от $y$ и $z$.

Пусть газ течет вдоль оси $O Y$ со скоростью $\vec{u}=(0, u(x), 0)$. Стационарное уравнение Больцмана в рассматриваемом одномерном случае имеет вид (см. [9], [10])

$$
s_{1} \frac{\partial f(x, \vec{s})}{\partial x}=\mathfrak{F}(f)
$$

где $\mathfrak{F}(f)$ - истинный интеграл столкновений.

Согласно модели Шахова точное уравнение Больцмана (1) заменяем приближенным (см. [3], [4]):

$$
s_{1} \frac{\partial f(x, \vec{s})}{\partial x}=\nu(x)\left[f_{0}(x, \vec{s})-f(x, \vec{s})\right]
$$

где

$$
f_{0}(x, \vec{s})=\frac{\rho(x)}{(\pi T(x))^{3 / 2}} e^{-(\vec{s}-\vec{u}(x))^{2} / T(x)}\left(1+\frac{\alpha s_{1} q(x)}{\rho(x) T^{2}(x)}\left(\frac{(\vec{s}-\vec{u}(x))^{2}}{T(x)}-\frac{5}{2}\right)\right) .
$$


Здесь $\alpha=4\left(1-P_{r}\right) / 5$, а $\rho(x), q(x), T(x)$ и $u(x)$ - плотность, проекция вектора потока $\vec{q}=(0, q(x), 0)$, температура и скорость газа соответственно, эти величины выражаются через функцию распределения:

$$
\begin{aligned}
\rho(x) & =\int f(x, \vec{s}) d^{3} s, \\
q(x) & =\int s_{1}(\vec{s}-\vec{u}(x))^{2} f(x, \vec{s}) d^{3} s, \\
\rho(x) T(x) & =\frac{2}{3} \int(\vec{s}-\vec{u}(x))^{2} f(x, \vec{s}) d^{3} s, \\
\rho(x) u(x) & =\int s_{2} f(x, \vec{s}) d^{3} s .
\end{aligned}
$$

Частота столкновений $\nu(x)$ задается выражением (см. [3], [9])

$$
\nu(x)=\int|g| \sigma f(x, \vec{s}) d^{3} s=\beta \int f(x, \vec{s}) d^{3} s,
$$

где $\sigma$ - сечение столкновений, $|g|$ - относительная скорость сталкивающихся частиц, $\beta$ - коэффициент пропорциональности, а также предполагалось, что $\sigma$ обратно пропорционально $|g|$ (псевдомаксвелловские молекулы), поэтому частота столкновений не зависит от скорости молекул. Следует отметить, что в общем случае частота столкновений зависит как от координат, так и от скорости молекул. В большинстве случаев считают, что она постоянна. Однако существуют многочисленные работы, в которых предполагается, что частота столкновений зависит либо только от координат (см., например, [3], [4]), либо только от модуля скорости молекул (см., например, [11]).

Заметим, что при $\alpha=0\left(P_{r}=1\right)$ в (3) модель Шахова переходит в модель БГК.

Введем функции распределения частиц $f^{+}(x, \vec{s})$ и $f^{-}(x, \vec{s})$, летящих к стенкам и отлетающих от них:

$$
\begin{aligned}
& f^{+}(x, \vec{s})=\left\{\begin{array}{lll}
f\left(x, s_{1}, s_{2}, s_{3}\right), & \text { если } & s_{1} \geqslant 0, \\
0, & \text { если } & s_{1}<0,
\end{array}\right. \\
& f^{-}(x, \vec{s})=\left\{\begin{array}{lll}
f\left(x,-s_{1}, s_{2}, s_{3}\right), & \text { если } & s_{1} \geqslant 0, \\
0, & \text { если } & s_{1}<0 .
\end{array}\right.
\end{aligned}
$$

С учетом (6) модельное уравнение Больцмана (2) перепишем в виде

$$
\pm s_{1} \frac{\partial f^{ \pm}(x, \vec{s})}{\partial x}+\nu(x) f^{ \pm}(x, \vec{s})=\nu(x) f_{0}(x, \vec{s}) .
$$

$\mathrm{K}$ уравнениям (7) добавим граничные условия

$$
f^{+}(0, \vec{s})=\frac{\rho_{+} e^{-|\vec{s}|^{2} / T_{+}}}{\left(\pi T_{+}\right)^{3 / 2}}, \quad f^{-}(d, \vec{s})=\frac{\rho_{-} e^{-|\vec{s}|^{2} / T_{-}}}{\left(\pi T_{-}\right)^{3 / 2}},
$$

где $\rho_{ \pm}-$плотности частиц, отлетающих от нижней и верхней стенок с температурами $T_{ \pm}$соответственно. 


\section{3. ПЕРЕХОД К НОВОМУ АРГУМЕНТУ. КИНЕТИЧЕСКАЯ ТОЛЩИНА СЛОЯ}

В уравнении (7) перейдем к новому аргументу $\tau$, который зависит от решения исходной задачи:

$$
d \tau=\nu(x) d x, \quad \tau(x)=\int_{0}^{x} \nu\left(x^{\prime}\right) d x^{\prime}=\beta \int_{0}^{x} \rho\left(x^{\prime}\right) d x^{\prime} .
$$

Введем следующие новые обозначения:

$$
\begin{gathered}
F(\tau(x), \vec{s})=f(x, \vec{s}), \quad F_{0}(\tau(x), \vec{s})=f_{0}(x, \vec{s}), \\
\varphi(\tau(x))=\rho(x), \quad \chi(\tau(x))=q(x), \quad \chi(\tau))=T(x), \\
\vec{v}(\tau(x))=\vec{u}(x) .
\end{gathered}
$$

Очевидно, что возврат к исходному аргументу $x$ осуществляется согласно

$$
x(\tau)=\int_{0}^{\tau} \frac{d \tau^{\prime}}{\beta \varphi\left(\tau^{\prime}\right)} .
$$

Из (9) и (10) следует, что $\tau(0)=x(0)=0$ и

$$
\begin{aligned}
& \tau(d) \equiv r=\beta \int_{0}^{d} \rho(x) d x, \\
& x(r)=d=\frac{1}{\beta} \int_{0}^{r} \frac{d \tau}{\varphi(\tau)} \equiv Q(r) .
\end{aligned}
$$

Заметим, что если $d<+\infty$, то $r<+\infty$. Величину $d$ назовем геометрической толщиной слоя, а величину $r$ - кинетической толщиной (см. [12]), эта величина неизвестна и подлежит дальнейшему определению.

В новых обозначениях граничная задача (7), (8) примет вид

$$
\begin{gathered}
\pm s_{1} \frac{\partial F^{ \pm}(\tau, \vec{s})}{\partial \tau}+F^{ \pm}(\tau, \vec{s})=F_{0}(\tau, \vec{s}) \\
F^{+}(0, \vec{s})=\frac{\rho_{+} e^{-|\vec{s}|^{2} / T_{+}}}{\left(\pi T_{+}\right)^{3 / 2}}, \quad F^{-}(r, \vec{s})=\frac{\rho_{-} e^{-|\vec{s}|^{2} / T_{-}}}{\left(\pi T_{-}\right)^{3 / 2}} .
\end{gathered}
$$

Как уже отмечалось, главным вопросом является определение кинетической толщины $r$ слоя. В граничной задаче $(13),(14)$ величина $r$ играет роль параметра.

После полного решения граничной задачи (13), (14) следует перейти к исходному аргументу $x$ по формуле (10), а с помощью соотношения (12) определить кинетическую толщину $r$ слоя по известной геометрической толщине $d$.

Из (12) имеем

$$
r=Q^{-1}(d),
$$

где $Q^{-1}$ является обратной для функции $Q$.

Существование обратной функции следует из изложенных ниже простых рассуждений. Так как $Q(0)=0, Q(r)>0$, то в силу непрерывности существует некоторая окрестность $\left[0, r_{0}\right]$, где $Q(r)$ сохраняет положительный знак, следовательно, $Q(r)$ возрастает на интервале $\left[0, r_{0}\right]$ и убывает на интервале $\left[r_{0},+\infty\right]$, принимая 
максимальное значение в точке $r_{0}$. Из монотонности функции $Q$ следует, что на каждом из интервалов $\left[0, r_{0}\right]$ и $\left[r_{0},+\infty\right)$ существует обратная функция $Q^{-1}$. Итак, имеет место

ЛЕмма 1. Пусть функиия $Q(r)$ задается согласно (12). Тогда существует обратная ей функиия $Q^{-1}$, а кинетическая толщина $r$ определяется согласно (15).

Отметим, что идея перехода к новому аргументу $\tau$, возникшая в нелинейной задаче переноса излучения в спектральных линиях, исходит из работ В. А. Амбарцумяна. Этот подход Амбарцумяном был назван методом самосогласованных оптических глубин (см. [13]). Метод определения реальной оптической толщины среды впервые был предложен Енгибаряном [14].

\section{4. ВЫВОД ОСНОВНЫХ НЕЛИНЕЙНЫХ ИНТЕГРАЛЬНЫХ УРАВНЕНИЙ}

После перехода к новому аргументу первоначальная нелинейная граничная задача $(7),(8)$ упростилась, но осталась нелинейной. С учетом граничных условий (14) из (13) имеем

$$
\begin{aligned}
& F^{+}(\tau, \vec{s})=\frac{\rho_{+} e^{-|\vec{s}|^{2} / T_{+}} e^{-\tau / s_{1}}}{\left(\pi T_{+}\right)^{3 / 2}}+\int_{0}^{\tau} e^{-(\tau-t) / s_{1}} F_{0}(t, \vec{s}) \frac{d t}{s_{1}} \\
& F^{-}(\tau, \vec{s})=\frac{\rho_{-} e^{-|\vec{s}|^{2} / T_{-}} e^{-(r-\tau) / s_{1}}}{\left(\pi T_{-}\right)^{3 / 2}}+\int_{\tau}^{r} e^{-(t-\tau) / s_{1}} F_{0}(t, \vec{s}) \frac{d t}{s_{1}},
\end{aligned}
$$

где

$$
F_{0}(\tau, \vec{s})=\frac{\varphi(\tau)}{(\pi \chi(\tau))^{3 / 2}} e^{-(\vec{s}-\vec{v}(\tau))^{2} / \chi(\tau)}\left(1+\frac{\alpha s_{1} \psi(\tau)}{\varphi(\tau) \chi^{2}(\tau)}\left(\frac{(\vec{s}-\vec{v}(\tau))^{2}}{\chi(\tau)}-\frac{5}{2}\right)\right)
$$

функции $\varphi, \psi, \chi, v$ являются моментами искомой функции распределения:

$$
\begin{aligned}
\varphi(\tau) & =\int\left[F^{+}(\tau, \vec{s})+F^{-}(\tau, \vec{s})\right] d s_{3} d s_{2} d s_{1}, \\
\psi(\tau) & =\int s_{1}(\vec{s}-\vec{v}(x))^{2}\left[F^{+}(\tau, \vec{s})+F^{-}(\tau, \vec{s})\right] d s_{3} d s_{2} d s_{1}, \\
\chi(\tau) \varphi(\tau) & =\frac{2}{3} \int(\vec{s}-\vec{v}(x))^{2}\left[F^{+}(\tau, \vec{s})+F^{-}(\tau, \vec{s})\right] d s_{3} d s_{2} d s_{1}, \\
v(\tau) \varphi(\tau) & =\int s_{2}\left[F^{+}(\tau, \vec{s})+F^{-}(\tau, \vec{s})\right] d s_{3} d s_{2} d s_{1}, \\
s_{1} & \in(0,+\infty), \quad s_{2}, s_{3} \in(-\infty,+\infty) .
\end{aligned}
$$

Подставляя (16) в (18) с учетом (17), проводя интегрирование и трудоемкие, но элементарные вычисления, получаем следующую систему нелинейных интегральных 
уравнений относительно искомых функций $\varphi, \psi, \chi, v$ :

$$
\begin{aligned}
\varphi(\tau) & =h_{1}(\tau)+\int_{0}^{r} W_{1}(\tau, t, \chi(t)) \varphi(t) d t+\int_{0}^{r} W_{2}(\tau, t, \chi(t)) \psi(t) d t \\
\psi(\tau) & =h_{2}(\tau)+\int_{0}^{r} W_{3}(\tau, t, \chi(t)) \varphi(t) d t+\int_{0}^{r} W_{4}(\tau, t, \chi(t)) \psi(t) d t, \\
\varphi(\tau) \chi(\tau) & =h_{3}(\tau)+\int_{0}^{r} W_{5}(\tau, t, \chi(t)) \varphi(t) d t+\int_{0}^{r} W_{6}(\tau, t, \chi(t)) \psi(t) d t \\
\varphi(\tau) v(\tau) & =\int_{0}^{r} W_{1}(\tau, t, \chi(t)) \varphi(t) v(t) d t+\int_{0}^{r} W_{4}(\tau, t, \chi(t)) \psi(t) v(t) d t
\end{aligned}
$$

где введены следующие обозначения:

$$
\begin{gathered}
h_{i}(\tau)=\int_{0}^{\infty}\left[e^{-\tau / s} G_{+}^{(i)}(s)+e^{-(r-\tau) / s} G_{-}^{(i)}(s)\right] d s, \quad i=1,2,3 \\
G_{ \pm}^{(1)}(s)=\frac{\rho_{ \pm}}{\sqrt{\pi T_{ \pm}}} e^{-s^{2} / T_{ \pm}}, \quad G_{ \pm}^{(2)}(s)=\frac{\rho_{ \pm} \sqrt{T_{ \pm}}}{\sqrt{\pi}} s e^{-s^{2} / T_{ \pm}}\left(\frac{s^{2}}{T_{ \pm}}+1+\frac{v^{2}}{T_{ \pm}}\right) \\
G_{ \pm}^{(3)}(s)=\frac{2}{3 s} G_{ \pm}^{(2)}(s)
\end{gathered}
$$

и

$$
\begin{aligned}
& W_{1}(\tau, t, \chi)=\frac{1}{\sqrt{\pi \chi}} \int_{0}^{\infty} e^{-|\tau-t| / s} e^{-s^{2} / \chi} \frac{d s}{s} \\
& W_{2}(\tau, t, \chi)=\frac{\alpha}{\sqrt{\pi \chi}} \int_{0}^{\infty} \frac{e^{-|\tau-t| / s} e^{-s^{2} / \chi}}{\chi^{3}}\left(s^{2}-\frac{3}{2} \chi\right) d s \\
& W_{3}(\tau, t, \chi)=\frac{\sqrt{\chi}}{\sqrt{\pi}} \int_{0}^{\infty} e^{-|\tau-t| / s} e^{-s^{2} / \chi}\left(\frac{s^{2}}{\chi}+1\right) d s \\
& W_{4}(\tau, t, \chi)=\frac{\alpha}{\sqrt{\pi \chi}} \int_{0}^{\infty} \frac{e^{-|\tau-t| / s} e^{-s^{2} / \chi}}{\chi^{3}} s\left(s^{4}-\frac{s^{2}}{2} \chi-\frac{\chi^{2}}{2}\right) d s, \\
& W_{5}(\tau, t, \chi)=\frac{2}{3} \frac{\sqrt{\chi}}{\sqrt{\pi}} \int_{0}^{\infty} e^{-|\tau-t| / s} e^{-s^{2} / \chi}\left(\frac{s^{2}}{\chi}+1\right) \frac{d s}{s}, \\
& W_{6}(\tau, t, \chi)=\frac{2 \alpha}{3 \sqrt{\pi \chi}} \int_{0}^{\infty} \frac{e^{-|\tau-t| / s} e^{-s^{2} / \chi}}{\chi^{3}}\left(s^{4}-\frac{s^{2}}{2} \chi-\frac{\chi^{2}}{2}\right) d s .
\end{aligned}
$$

Ниже мы будем решать нелинейную систему (19)-(22). Интегральный оператор указанной системы не обладает свойством монотонности, что весьма усложняет построение неподвижной точки. Однако структура системы специфична. Во-первых, заметим, что при любом заданном виде функций $\varphi, \psi, \chi$ уравнение $(22)$ обладает тривиальным решением. Это следует также из физических соображений, так как пластинки неподвижны. Во-вторых, при любом заданном виде функции $\chi$ уравнения (19), (20) образуют линейную систему интегральных уравнений относительно $\varphi$ и $\psi$. Наконец, в-третьих, при заданных $\varphi$ и $\psi$ уравнение $(21)$ представляет собой нелинейное скалярное интегральное уравнение относительно функции $\chi$. Изложенные выше соображения наводят на мысль предложить следующую схему решения системы (19)-(22). Сначала фиксируем тривиальное решение уравнения (22). Далее 
рассмотрим систему линейных интегральных уравнений (19), (20) в предположении постоянства температуры $\chi$, полагая $\chi \equiv 1$. Тогда из (19) и (20) имеем

$$
\begin{aligned}
& \varphi(\tau)=h_{1}(\tau)+\int_{0}^{r} K_{11}(\tau-t) \varphi(t) d t+\int_{0}^{r} K_{12}(\tau-t) \psi(t) d t \\
& \psi(\tau)=h_{2}(\tau)+\int_{0}^{\infty} K_{21}(\tau-t) \varphi(t) d t+\int_{0}^{r} K_{22}(\tau-t) \psi(t) d t
\end{aligned}
$$

где

$$
\begin{gathered}
K_{i j}(\tau)=\int_{0}^{\infty} e^{-|\tau| / s} G_{i j}(s) d s, \quad i, j=1,2 \\
G_{11}(s)=\frac{1}{\sqrt{\pi} s} e^{-s^{2}}, \quad G_{12}(s)=\frac{\alpha}{\sqrt{\pi}} e^{-s^{2}}\left(s^{2}-\frac{3}{2}\right), \\
G_{21}(s)=\frac{1}{\sqrt{\pi}} e^{-s^{2}}\left(s^{2}+1\right), \quad G_{22}(s)=\frac{\alpha}{\sqrt{\pi}}\left(s^{5}-\frac{s^{3}}{2}-\frac{s}{2}\right),
\end{gathered}
$$

a $h_{i}(\tau), i=1,2$, задается согласно (23), (24).

Исходя из физических рассуждений упростим уравнение (21). Заметим, что второй интеграл в уравнении (21) содержит в себе вектор потока энергии. В приближении модели БГК имеем $W_{6}=0$, так как при этом $\alpha=0\left(P_{r}=1\right)$. Поэтому функции $\chi(\tau)$ и $\psi(\tau)$, входящие во второй интеграл в уравнении $(21)$, заменим, например, их значениями в свободномолекулярном режиме (см. [7]):

$$
\begin{gathered}
\chi(\tau) \equiv T_{0}, \quad \psi(\tau) \equiv q_{0}, \\
T_{0}=\sqrt{T_{+} T_{-}}, \quad q_{0}=\frac{\sqrt{T_{+}}}{\sqrt{\pi}} \rho_{+}\left(T_{+}-T_{-}\right), \quad q_{0}>0, \quad T_{+}>T_{-} .
\end{gathered}
$$

Для простоты примем $T_{0} \equiv 1$. Тогда уравнение $(21)$ преобразуется к нелинейному уравнению типа Урысона:

$$
\varphi(\tau) \chi(\tau)=h(\tau)+\int_{0}^{r} W_{5}(\tau, t, \chi(t)) \varphi(t) d t
$$

где

$$
\begin{aligned}
h(\tau) & =\frac{2 \alpha q_{0}}{3 \sqrt{\pi}}+\int_{0}^{\infty}\left[e^{-\tau / s} G_{+}(s)+e^{-(r-\tau) / s} G_{-}(s)\right] d s \\
G_{ \pm}(s) & =\frac{2}{3 \sqrt{\pi}} \rho_{ \pm} \sqrt{T_{ \pm}} e^{-s^{2} / T_{ \pm}}\left(\frac{s^{2}}{T_{ \pm}}+1\right)-\frac{2 \alpha q_{0}}{3 \sqrt{\pi}} e^{-s^{2}}\left(s^{5}-\frac{s^{3}}{2}-\frac{s}{2}\right) .
\end{aligned}
$$

В разделе 7 будет рассмотрена линейная система интегральных уравнений относительно $\varphi, \psi$ и $\chi$, получаемая в результате линеаризации исходной системы (19)-(21).

В результате численного решения нелинейной системы (19)-(21) предложенным нами подходом и решения соответствующей линеаризованной системы найдены температурные скачки $\left|\chi(0)-T_{+}\right|$и $\left|\chi(r)-T_{-}\right|$как в нелинейном, так и в линейном случае. Оказывается, что они мало различаются. Последнее обстоятельство может служить доводом для обоснования (на эвристическом уровне) изложенного выше подхода приближенного решения нелинейной системы (19)-(21). 


\section{5. О РАЗРЕШИМОСТИ ЛИНЕЙНОЙ СИСТЕМЫ ИНТЕГРАЛЬНЫХ УРАВНЕНИЙ (26)}

Настоящий раздел посвящен изучению и решению системы (26).

Пусть $E$ - одно из следующих банаховых пространств: $L_{p}[0, r], p \geqslant 1, M[0, r]$. Обозначим через $E^{2}=E \times E$ пространство двумерных вектор-столбцов с элементами из $E$, а $\widehat{K}$ - матричный интегральный оператор

$$
(\widehat{K} f)(x)=\int_{0}^{r} K(x-t) f(t) d t .
$$

Введем вектор-функцию $\zeta=(\varphi, \psi)^{\mathrm{T}}$, где $\mathrm{T}$ - знак транспонирования. Рассмотрим следующее операторное уравнение:

$$
\zeta=\widehat{K} \eta
$$

В любом из пространств $E^{2}$ имеет место оценка

$$
\|\zeta\|_{E^{2}} \leqslant \max (\lambda(r), \mu(r))\|\eta\|_{E^{2}}=\sigma\|\eta\|_{E^{2}},
$$

где

$$
\begin{aligned}
& \lambda(r) \equiv\left\|\widehat{K}_{11}\right\|_{L_{1}}+\left\|\widehat{K}_{12}\right\|_{L_{1}} \geqslant\left\|\widehat{K}_{11}\right\|_{E}+\left\|\widehat{K}_{12}\right\|_{E}, \\
& \mu(r) \equiv\left\|\widehat{K}_{21}\right\|_{L_{1}}+\left\|\widehat{K}_{22}\right\|_{L_{1}} \geqslant\left\|\widehat{K}_{21}\right\|_{E}+\left\|\widehat{K}_{22}\right\|_{E} .
\end{aligned}
$$

Действительно,

$$
\begin{aligned}
\|\zeta\|_{E^{2}} & \leqslant\|\widehat{K} \eta\|_{E^{2}}=\left\|\widehat{K}_{11} \eta_{1}+\widehat{K}_{12} \eta_{2}\right\|_{E}+\left\|\widehat{K}_{21} \eta_{1}+\widehat{K}_{22} \eta_{2}\right\|_{E} \leqslant \\
& \leqslant\left(\left\|\widehat{K}_{11}\right\| \widehat{K}_{E}+\left\|\widehat{K}_{21}\right\|_{E}\right)\left\|\eta_{1}\right\|_{E}+\left(\left\|\widehat{K}_{12}\right\|_{E}+\left\|\widehat{K}_{22}\right\|_{E}\right)\left\|\eta_{2}\right\|_{E} \leqslant \\
& \leqslant \max (\lambda(r), \mu(r)) \cdot\|\eta\|_{E^{2}} .
\end{aligned}
$$

Из (26), (27) следует

$$
\begin{aligned}
\lambda(r)= & \int_{-r}^{r} K_{11}(\tau) d \tau+\int_{-r}^{r}\left|K_{12}(\tau)\right| d \tau=1-\frac{2}{\sqrt{\pi}} \int_{0}^{\infty} e^{-r / s} e^{-s^{2}} d s+ \\
& +\frac{2 \alpha}{\sqrt{\pi}} \int_{0}^{\infty}\left(1-e^{-r / s}\right) s\left|s^{2}-\frac{3}{2}\right| d s \\
\mu(r)= & \int_{-r}^{r} K_{21}(\tau) d \tau+\int_{-r}^{r}\left|K_{22}(\tau)\right| d \tau=\frac{2}{\sqrt{\pi}}-\frac{2}{\sqrt{\pi}} \int_{0}^{\infty} e^{-r / s} e^{-s^{2}}\left(s^{3}+s\right) d s+ \\
& +\frac{2 \alpha}{\sqrt{\pi}} \int_{0}^{\infty} e^{-s^{2}}\left(1-e^{-r / s}\right) s^{2}\left|s^{4}-\frac{s^{2}}{2}-\frac{1}{2}\right| d s
\end{aligned}
$$

Заметим, что $\lambda(0)=0, \mu(0)=0, \lambda(r)>0, \mu(r)>0$, функция $\lambda(r)$ возрастает по $r$, функция $\mu(r)$ возрастает по $r$, следовательно, существуют $r_{1}$ и $r_{2}$, при которых $\lambda\left(r_{1}\right)<1, \mu\left(r_{2}\right)<1$. Выбираем $r_{m}=\min \left(r_{1}, r_{2}\right)$. Тогда имеем

$$
\sigma=\max \left(\lambda\left(r_{m}\right), \mu\left(r_{m}\right)\right)<1,
$$

и интегральный оператор исходной системы (26) является сжимающим с коэффициентом сжатия $\sigma<1$. 
Решение системы $(\varphi, \psi)^{\mathrm{T}}$ строится с помощью следующих итераций:

$$
\begin{aligned}
\varphi^{(n+1)}(\tau) & =h_{1}(\tau)+\int_{0}^{r} K_{11}(\tau-t) \varphi^{(n)}(t) d t+\int_{0}^{r} K_{12}(\tau-t) \psi^{(n)}(t) d t \\
\psi^{(n+1)}(\tau) & =h_{2}(\tau)+\int_{0}^{\infty} K_{21}(\tau-t) \varphi^{(n)}(t) d t+\int_{0}^{r} K_{22}(\tau-t) \psi^{(n)}(t) d t, \\
\varphi^{(0)} & =0, \quad \psi^{(0)}=0, \quad n=1,2,3, \ldots
\end{aligned}
$$

Итак, имеет место

Теорема 1. Пусть ядра $K_{i j}(\tau), i, j=1,2$, системы уравнений (26) задаются согласно (27). Тогда система уравнений (26) имеет единственное решение в пространстве $E^{2}[0, r]$. Решением является предел итераций (38).

ЗАмЕчАниЕ 1. Численные расчеты показали, что $\lambda(r)<1$ при $r_{1}=0.85, \mu(r)<1$ при $r_{2}=0.9$, т. е. $r_{m}=0.85$. Тогда $\lambda\left(r_{1}\right)=0.991, \mu\left(r_{2}\right)=0.984$, т. е. коэффициент сжатия $\sigma=0.991$.

\section{6. О РАЗРЕШИМОСТИ НЕЛИНЕЙНОГО УРАВНЕНИЯ (29)}

В настоящем разделе мы исследуем вопрос о разрешимости уравнения (29) при заданной непрерывной на интервале $[0, r]$ функции $\varphi(\tau)$. Сначала убедимся, что функция $W_{5}(\tau, t, z)$ монотонна по третьему аргументу. Рассмотрим функцию

$$
\sqrt{z} e^{-s^{2} / z}\left(\frac{s^{2}}{z}+1\right), \quad z \in[0,+\infty), \quad s>0 .
$$

Легко убедиться, что ее производная положительна для произвольного $s^{2}>0$. Следовательно, функция $W_{5}$ возрастает по $z$. Теперь покажем, что $h(\tau) \geqslant 0$. Из (30) имеем

$$
h(\tau)=h_{3}(\tau)+\frac{2 \alpha q_{0}}{3 \sqrt{\pi}} \xi(\tau),
$$

где $h_{3}(\tau)$ задается согласно $(23)$, а

$$
\xi(\tau)=1-\int_{0}^{\infty}\left[e^{-\tau / s}+e^{-(r-\tau) / s}\right] s e^{-s^{2}}\left(s^{4}-\frac{s^{2}}{2}-\frac{1}{2}\right) d s
$$

откуда имеем

$$
\begin{aligned}
\xi(\tau)=1 & +\int_{0}^{1}\left[e^{-\tau / s}+e^{-(r-\tau) / s}\right] s e^{-s^{2}}\left(s^{2}+\frac{1}{2}\right)\left(1-s^{2}\right) d s- \\
& -\int_{1}^{\infty}\left[e^{-\tau / s}+e^{-(r-\tau) / s}\right] s e^{-s^{2}}\left(s^{2}+\frac{1}{2}\right)\left(s^{2}-1\right) d s
\end{aligned}
$$

Наименьшее значение функции $e^{-\tau / s}+e^{-(r-\tau) / s}$ на отрезке $[0, r]$ при каждом фиксированном $s$ равно $2 e^{-r / 2 s}$, а наибольшее значение $-1+e^{-r / s}$. Тогда из (41) получим $\xi(\tau) \geqslant 1+2 \int_{0}^{1} e^{-r / 2 s} e^{-s^{2}} s\left(1-s^{2}\right)\left(s^{2}+\frac{1}{2}\right) d s-\int_{1}^{\infty}\left(1+e^{-r / s}\right) e^{-s^{2}} s\left(s^{2}-1\right)\left(s^{2}+1\right) d s$, при $r=0.85$ (см. замечание 1 ) имеем $\xi(\tau) \geqslant 0.079$. Поскольку $h_{3}(\tau) \geqslant 0$, то $h(\tau) \geqslant 0$. 
Рассмотрим следующие итерации для уравнения (29):

$$
\begin{aligned}
\varphi(\tau) \chi^{(n+1)}(\tau) & =h(\tau)+\int_{0}^{r} W_{5}\left(\tau, t, \chi^{(n)}(t)\right) \varphi(t) d t, \\
\chi^{(0)}(\tau) & =\frac{h(\tau)}{\varphi(\tau)}, \quad \tau \in[0, r], \quad n=0,1,2, \ldots .
\end{aligned}
$$

Интегрируя уравнение (42) по $\tau$ от 0 до $r$ и учитывая выражение для $W_{5}(\tau, t, \chi)$ из $(25)$ и монотонность функции $W_{5}$, получим

$$
\begin{aligned}
\int_{0}^{r} \varphi(\tau) \chi^{(n+1)}(\tau) d \tau & \leqslant \int_{0}^{r} h(\tau) d \tau+\int_{0}^{r} \int_{0}^{r} W_{5}\left(\tau, t, \chi^{(n+1)}(t)\right) \varphi(t) d t d \tau= \\
& =\int_{0}^{r} h(\tau) d \tau+\int_{0}^{r} \varphi(t) \chi^{(n+1)}(t)\left[1-\Gamma\left(t, \chi^{(n+1)}(t)\right)\right] d t
\end{aligned}
$$

где

$$
\Gamma(t, z)=\int_{0}^{r} W_{5}(\tau, t, z) d \tau=\frac{2}{3 \sqrt{\pi z}} \int_{0}^{\infty} e^{-s^{2} / z}\left(\frac{s^{2}}{z}+1\right)\left[e^{-t / s}+e^{-(r-t) / s}\right] d s .
$$

Из (43) имеем

$$
\int_{0}^{r} \varphi(\tau) \chi^{(n+1)}(t) \chi\left(t, \chi^{(n+1)}(t)\right) d t \leqslant C_{r}
$$

где

$$
0<C_{r}=\int_{0}^{r} h(\tau) d \tau=\frac{2 \alpha q_{0}}{3 \sqrt{\pi}} r+\int_{0}^{\infty} s\left(G_{+}(s)+G_{-}(s)\right)\left(1-e^{-r / s}\right) d s .
$$

Функция $\Gamma(t, z)$ непрерывна по совокупности своих аргументов, а из (44) следует, что $z \Gamma(t, z)$ возрастает по $z$. В силу того, что $z \Gamma(t, z)$ возрастает по $z, \chi^{(n+1)}(\tau) \geqslant \chi^{(n)}(\tau)$, имеем

$$
\varphi(\tau) \chi^{(n+1)}(\tau) \Gamma\left(\tau, \chi^{(n+1)}(\tau)\right) \geqslant \varphi(\tau) \chi^{(n)}(\tau) \Gamma\left(\tau, \chi^{(n)}(\tau)\right) .
$$

Из (45) и (46) следует

$$
\int_{0}^{r} \varphi(\tau) \chi^{(n)}(\tau) \Gamma\left(\tau, \chi^{(n)}(\tau)\right) d \tau \leqslant C_{r}
$$

Согласно указанным выше свойствам функций $\Gamma(t, z)$ и $z \Gamma(t, z)$ и из $(47)$ следует существование поточечного предела функциональной последовательности $\left\{\chi^{(n)}(\tau)\right\}_{n=0}^{\infty}$, $\lim _{n \rightarrow \infty} \chi^{(n)}(\tau)=\chi(\tau)$, причем

$$
\int_{0}^{r} \varphi(\tau) \chi(\tau) \Gamma(\tau, \chi(\tau)) d \tau \leqslant C_{r}
$$

Теорема 2. Пусть $\varphi(\tau) \in C[0, r]$ - заданная непрерывная функция, удовлетворяющая системе (26). Тогда уравнение (29) имеет положительное решение. Имеют место оченки

$$
\int_{0}^{r} \chi(t) \Gamma(t, \chi(t)) d t \leqslant \frac{C_{r}}{m}, \quad \chi(t) \geqslant \frac{h(\tau)}{M},
$$

¿əe

$$
m=\min _{t \in[0, r]} \varphi(t), \quad M=\max _{t \in[0, r]} \varphi(t)
$$




\section{7. ЛИНЕАРИЗАЦИЯ ИСХОДНОЙ НЕЛИНЕЙНОЙ СИСТЕМЫ ИНТЕГРАЛЬНЫХ УРАВНЕНИЙ (19)-(21)}

Настоящий раздел посвящен рассмотрению линейного приближения первоначальной нелинейной системы (19)-(21). В линейном приближении искомые функции $\varphi, \psi$ и $\chi$ представимы в виде

$$
\varphi(\tau)=1+\Delta \varphi(\tau), \quad \psi(\tau)=q_{0}+\Delta \psi(\tau), \quad \chi(\tau)=1+\Delta \chi(\tau),
$$

где $\Delta \varphi, \Delta \psi, \Delta \chi$ - возмущения плотности, потока энергии и температуры газа соответственно.

Линеаризуя функции $W_{i}(\tau, t, z), i=1,2, \ldots, 6$, по $z$ в окрестности нуля и ограничиваясь первыми двумя членами разложения, получим

$$
\begin{aligned}
& W_{1}(\tau, t, \chi) \approx \frac{1}{\sqrt{\pi}} \int_{0}^{\infty} e^{-|\tau-t| / s} e^{-s^{2}}\left[1+\left(s^{2}-\frac{1}{2}\right) \Delta \chi(t)\right] \frac{d s}{s}, \\
& W_{2}(\tau, t, \chi) \approx \frac{\alpha}{\sqrt{\pi}} \int_{0}^{\infty} e^{-|\tau-t| / s} e^{-s^{2}}\left[\left(s^{2}-\frac{3}{2}\right)+\left(s^{4}-5 s^{2}+\frac{15}{4}\right) \Delta \chi(t)\right] d s, \\
& W_{3}(\tau, t, \chi) \approx \frac{1}{\sqrt{\pi}} \int_{0}^{\infty} e^{-|\tau-t| / s} e^{-s^{2}}\left[s^{2}+1+\left(s^{4}+\frac{s^{2}}{2}+\frac{1}{2}\right) \Delta \chi(t)\right] d s, \\
& W_{4}(\tau, t, \chi) \approx \frac{\alpha}{\sqrt{\pi}} \int_{0}^{\infty} e^{-|\tau-t| / s} e^{-s^{2}} s\left[s^{4}-\frac{s^{2}}{2}-\frac{1}{2}+\left(s^{6}-4 s^{4}+\frac{3}{4} s^{2}+\frac{3}{4}\right) \Delta \chi(t)\right] d s, \\
& W_{5}(\tau, t, \chi) \approx \frac{2}{3 \sqrt{\pi}} \int_{0}^{\infty} e^{-|\tau-t| / s} e^{-s^{2}}\left[s^{2}+1+\left(s^{4}+\frac{s^{2}}{2}+\frac{1}{2}\right) \Delta \chi(t)\right] \frac{d s}{s}, \\
& W_{6}(\tau, t, \chi) \approx \frac{2 \alpha}{3 \sqrt{\pi}} \int_{0}^{\infty} e^{-|\tau-t| / s} e^{-s^{2}}\left[s^{4}-\frac{s^{2}}{2}-\frac{1}{2}+\left(s^{6}-4 s^{4}+\frac{3}{4} s^{2}+\frac{3}{4}\right) \Delta \chi(t)\right] d s .
\end{aligned}
$$

Подставляя эти выражения в (19)-(21), с учетом (49) и проделывая несколько громоздких, но несложных выкладок, приходим к следующей системе линейных интегральных уравнений относительно поправок $\Delta \varphi, \Delta \psi, \Delta \chi$ :

$$
\begin{aligned}
\Delta \varphi(\tau)= & g_{1}(\tau)+\int_{0}^{r} K_{11}(\tau-t) \Delta \varphi(t) d t+ \\
& +\int_{0}^{r} K_{12}(\tau-t) \Delta \psi(t) d t+\int_{0}^{r} K_{13}(\tau-t) \Delta \chi(t) d t \\
\Delta \psi(\tau)= & g_{2}(\tau)+\int_{0}^{r} K_{21}(\tau-t) \Delta \varphi(t) d t+ \\
& +\int_{0}^{r} K_{22}(\tau-t) \Delta \psi(t) d t+\int_{0}^{r} K_{23}(\tau-t) \Delta \chi(t) d t \\
\Delta \chi(\tau)= & g_{3}(\tau)+\int_{0}^{r} K_{31}(\tau-t) \Delta \varphi(t) d t+ \\
+ & \int_{0}^{r} K_{32}(\tau-t) \Delta \psi(t) d t+\int_{0}^{r} K_{33}(\tau-t) \Delta \chi(t) d t
\end{aligned}
$$

где

$$
K_{i j}(\tau)=\int_{0}^{\infty} e^{-|\tau| / s} G_{i j}(s) d s, \quad i, j=1,2,3,
$$


и

$$
\begin{aligned}
& G_{11}(s)=\frac{1}{\sqrt{\pi} s} e^{-s^{2}}, \quad G_{12}(s)=\frac{\alpha}{\sqrt{\pi}} e^{-s^{2}}\left(s^{2}-\frac{3}{2}\right), \\
& G_{13}(s)=\frac{1}{\sqrt{\pi}} e^{-s^{2}}\left[\left(s^{2}-\frac{1}{2}\right) \frac{1}{s}+\alpha q_{0}\left(s^{4}-5 s^{2}+\frac{15}{4}\right)\right], \\
& G_{21}(s)=\frac{1}{\sqrt{\pi}} e^{-s^{2}}\left(s^{2}+1\right), \quad G_{22}(s)=\frac{\alpha}{\sqrt{\pi}} e^{-s^{2}}\left(s^{5}-\frac{s^{3}}{2}-\frac{s}{2}\right), \\
& G_{23}(s)=\frac{1}{\sqrt{\pi}} e^{-s^{2}}\left[\left(s^{4}+\frac{s^{2}}{2}+\frac{1}{2}\right)+\alpha q_{0}\left(s^{7}-4 s^{5}+s^{4}+\frac{3}{4} s^{3}+\frac{s^{2}}{2}+\frac{3}{4} s+\frac{1}{2}\right)\right] \text {, } \\
& G_{31}(s)=\frac{2}{3 \sqrt{\pi} s} e^{-s^{2}}\left(s^{2}-\frac{1}{2}\right), \quad G_{32}(s)=\frac{2 \alpha}{3 \sqrt{\pi}} e^{-s^{2}}\left(s^{4}-2 s^{2}+\frac{7}{4}\right), \\
& G_{33}(s)=\frac{2}{3 \sqrt{\pi} s} e^{-s^{2}}\left[\left(s^{2}-\frac{1}{2}\right)^{2}+1\right]+\frac{2 \alpha q_{0}}{3 \sqrt{\pi}} e^{-s^{2}}\left[s^{6}-\frac{11}{2} s^{4}+\frac{33}{4} s^{2}-\frac{39}{8}\right], \\
& g_{1}(\tau)=h_{1}(\tau)-\frac{\alpha q_{0}}{2 \sqrt{\pi}}-\frac{1}{\sqrt{\pi}} \int_{0}^{\infty}\left[e^{-\tau / s}+e^{-(r-\tau) / s}\right] e^{-s^{2}} \times \\
& \times\left[1+\alpha q_{0}\left(s^{3}-\frac{s}{2}\right)\right] d s, \\
& g_{2}(\tau)=h_{2}(\tau)+\frac{2}{\sqrt{\pi}}-q_{0}+\frac{5 \alpha q_{0}}{4}-\frac{1}{\sqrt{\pi}} \int_{0}^{\infty}\left[e^{-\tau / s}+e^{-(r-\tau) / s}\right] e^{-s^{2}} \times \\
& \times\left[s^{3}+s+\alpha q_{0}\left(s^{6}-\frac{s^{4}}{2}-\frac{s^{2}}{2}\right)\right] d s, \\
& g_{3}(\tau)=h_{3}(\tau)-g_{1}(\tau)+\frac{2 \alpha q_{0}}{3 \sqrt{\pi}}-\frac{2}{3 \sqrt{\pi}} \int_{0}^{\infty}\left[e^{-\tau / s}+e^{-(r-\tau) / s}\right] e^{-s^{2}} \times \\
& \times\left[s^{2}+1+\alpha q_{0}\left(s^{5}-\frac{s^{3}}{2}-\frac{s}{2}\right)\right] d s,
\end{aligned}
$$

а функции $h_{i}(\tau), i=1,2,3$, задаются согласно (23).

В частном случае, когда $\alpha=0\left(P_{r}=1\right), \psi \equiv 0$ (модель БГК), система (50) упрощается и преобразовывается в следующую систему:

$$
\begin{aligned}
& \Delta \varphi(\tau)=\bar{g}_{1}(\tau)+\int_{0}^{r} K_{11}(\tau-t) \Delta \varphi(t) d t+\int_{0}^{r} \bar{K}_{13}(\tau-t) \Delta \chi(t) d t \\
& \Delta \chi(\tau)=\bar{g}_{3}(\tau)+\int_{0}^{r} K_{31}(\tau-t) \Delta \varphi(t) d t+\int_{0}^{r} \bar{K}_{33}(\tau-t) \Delta \chi(t) d t
\end{aligned}
$$

где

$$
\begin{aligned}
\bar{g}_{1}(\tau) & =h_{1}(\tau)-\frac{1}{\sqrt{\pi}} \int_{0}^{\infty}\left[e^{-\tau / s}+e^{-(r-\tau) / s}\right] e^{-s^{2}} d s \\
\bar{g}_{3}(\tau) & =h_{3}(\tau)-\bar{g}_{1}(\tau)-\frac{2}{3 \sqrt{\pi}} \int_{0}^{\infty}\left[e^{-\tau / s}+e^{-(r-\tau) / s}\right]\left(s^{2}+1\right) e^{-s^{2}} d s, \\
K_{31} & =\frac{2}{3} \bar{K}_{13}, \quad \bar{K}_{13}(\tau)=\frac{1}{\sqrt{\pi}} \int_{0}^{\infty} e^{-|\tau| / s} e^{-s^{2}}\left(s^{2}-\frac{1}{2}\right) \frac{d s}{s} \\
\bar{K}_{33} & =\frac{2}{3 \sqrt{\pi}} \int_{0}^{\infty} e^{-|\tau| / s} e^{-s^{2}}\left[\left(s^{2}-\frac{1}{2}\right)^{2}+1\right] \frac{d s}{s} .
\end{aligned}
$$

Система уравнений (52) была изучена в работе [15]. 


\section{8. РЕЗУЛЬТАТЫ НЕКОТОРЫХ ЧИСЛЕННЫХ РАСЧЕТОВ}

В настоящем разделе приводятся результаты некоторых численных расчетов. Найдены значения температурного скачка как в линейном, так и в нелинейном случае. Осуществляется сравнительный анализ между моделью БГК и моделью Шахова.

Итак, сначала находим максимальное значение $r$, при котором система (50) однозначно разрешима.

Обозначим

$$
\lambda_{i}(r)=\sum_{j=1}^{3} \int_{-r}^{r}\left|K_{i j}(\tau)\right| d \tau, \quad i=1,2,3 .
$$

Из (51) имеем

$$
\begin{aligned}
\lambda_{1}(r)= & \frac{2}{\sqrt{\pi}} \int_{0}^{\infty} e^{-s^{2}}\left(1-e^{-r / s}\right) \times \\
& \times\left(1+\alpha s\left|s^{2}-\frac{3}{2}\right|+\left|s^{2}-\frac{1}{2}+\alpha q_{0}\left(s^{5}-5 s^{3}+\frac{15}{4} s\right)\right|\right) d s, \\
\lambda_{2}(r)= & \frac{2}{\sqrt{\pi}} \int_{0}^{\infty} e^{-s^{2}}\left(1-e^{-r / s}\right)\left[s^{3}+s+\alpha\left|s^{6}-\frac{s^{4}}{2}-\frac{s^{2}}{2}\right|+\right. \\
& \left.+\left(s^{5}+\frac{s^{3}}{2}+\frac{s}{2}\right)+\alpha q_{0}\left|s^{8}-4 s^{6}+s^{5}+\frac{3}{4} s^{4}+\frac{s^{3}}{2}+\frac{3}{4} s^{2}+\frac{s}{2}\right|\right] d s, \\
\lambda_{3}(r)= & \frac{4}{3 \sqrt{\pi}} \int_{0}^{\infty} e^{-s^{2}}\left(1-e^{-r / s}\right)\left[\left|s^{2}-\frac{1}{2}\right|+\alpha\left|s^{5}-2 s^{3}+\frac{7}{4} s\right|+\right. \\
& \left.+\left(s^{2}-\frac{1}{2}\right)^{2}+1+\alpha q_{0}\left|s^{7}-\frac{11}{2} s^{5}+\frac{33}{4} s^{3}-\frac{39}{8} s\right|\right] d s .
\end{aligned}
$$

При выводе линейных уравнений (50) мы приняли $T_{0}=\sqrt{T_{+} T_{-}}=1, \rho_{0}=\left(\rho_{+}+\right.$ $\left.\rho_{-}\right) / 2=1$. Из условия непротекания $\rho_{+} \sqrt{T_{+}}=\rho_{-} \sqrt{T_{-}}$следует, что $\rho_{+}=2 /\left(1+T_{+}\right)$, $\rho_{-}=2 T_{+} /\left(1+T_{+}\right)$. Для проекции вектора потока имеем

$$
q_{0}=\frac{\rho_{+} \sqrt{T_{+}}}{\sqrt{\pi}}\left(T_{+}-T_{-}\right)=\frac{2\left(T_{+}-1\right)}{\sqrt{\pi T_{+}}} .
$$

Так как для одноатомных газов число Прандтля равно $2 / 3$, то примем $\alpha=(4 / 5)(1-$ $\left.P_{r}\right)=8 / 15$. Численные расчеты выполнены для значений

$$
T_{+}=2, \quad T_{-}=\frac{1}{2}, \quad q_{0}=\sqrt{\frac{2}{\pi}}, \quad \rho_{0}=1 .
$$

Численные расчеты показывают, что коэффициент сжатия

$$
\sigma=\max \left(\lambda_{1}\left(r_{m}\right), \lambda_{2}\left(r_{m}\right), \lambda_{3}\left(r_{m}\right)\right)=0.998
$$

становится меньше единицы при $r \leqslant 0.32$. Это означает, что система (50) при $r \leqslant 0.32$ имеет единственное решение.

Система уравнений (50) решается с помощью итераций. Полученную тройку $(1+\Delta \varphi),\left(q_{0}+\Delta \psi\right),(1+\Delta \chi)$ назовем решением исходной системы (19)-(21) в линейном приближении. 
Далее итерациями решается система (26) и нелинейное уравнение (42). Таким путем построенное решение $(\varphi(\tau), \psi(\tau), \chi(\tau))$ назовем приближенным решением нелинейной системы (19)-(21).

В нелинейном случае в результате решения системы (26) и уравнения (42) в рамках модели Шахова для температурного скачка (см. [16]) на нижней и верхней стенках при $r=0.2, T_{+}=2, T_{-}=1 / 2, q_{0}=\sqrt{2 / \pi}$ получены следующие значения с точностью до постоянного множителя:

$$
\varkappa_{T}^{+} \sim\left|\chi_{\text {nonlin }}(0)-T_{+}\right|=0.69, \quad \varkappa_{T}^{-} \sim\left|\chi_{\text {nonlin }}(r)-T_{-}\right|=0.34
$$

В линейном приближении в результате решения системы (50) получены следующие значения:

$$
\varkappa_{T}^{+} \sim\left|\chi_{\operatorname{lin}}(0)-T_{+}\right|=0.711, \quad \varkappa_{T}^{-} \sim\left|\chi_{\operatorname{lin}}(r)-T_{-}\right|=0.299 .
$$

Для сравнения приведем также результаты численных расчетов, полученные в рамках модели БГК в линейном и нелинейном случаях при $T_{+}=2, r=0.2, \chi=0$ (см. [15]):

$$
\begin{array}{ll}
\varkappa_{T}^{+} \sim\left|\chi_{\operatorname{nonlin}}(0)-T_{+}\right|=0.79, & \varkappa_{T}^{-} \sim\left|\chi_{\text {nonlin }}(r)-T_{-}\right|=0.403, \\
\varkappa_{T}^{+} \sim\left|\chi_{\operatorname{lin}}(0)-T_{+}\right|=0.821, & \varkappa_{T}^{-} \sim\left|\chi_{\operatorname{lin}}(r)-T_{-}\right|=0.394 .
\end{array}
$$

Относительная ошибка в линейном и нелинейном случаях в рамках модели Шахова незначительна, что дает основание на эвристическом уровне утверждать, что предложенный нами подход для нахождения приближенного (поэтапного) решения нелинейной системы (19)-(21) близок к точному решению.

Чтобы сравнить численные расчеты с результатами других авторов, необходимо для заданного $r$ решить систему (26) и уравнение (42), совершить переход к старому аргументу $x$ согласно $(10)$ и, наконец, определить геометрическую толщину слоя $d$ согласно (12), тем самым установить по заданной кинетической толщине $r$, какая геометрическая толщина $d$ ей соответствует. Однако эти вопросы выходят за рамки настоящей статьи, им будет посвящена отдельная работа.

Благодарности. Авторы выражают глубокую благодарность рецензенту за полезные замечания.

\section{Список литературы}

[1] P. L. Bhatnagar, E. P. Gross, M. Krook, "A model for collision processes in gases", Phys. Rev., 94:3 (1954), 511-525.

[2] L. H. Holway, "New statistical model for kinetic theory: methods of construction", Phys. Fluids, 9:9 (1966), 1658-1673.

[3] Е. М. Шахов, "Об обобщении релаксационного кинетического уравнения Крука", Изв. АН СССР. Сер. МЖГ, 1968, № 5, 142-145.

[4] V. A. Titarev, "Conservative numerical methods for advanced model kinetic equations", European Conference on Computational Fluid Dynamics (TU Delft, The Netherlands, 2006), eds. E. Oñate, P. Wessling, J. Périaux, Delft University of Technology, Netherlands, 2006, 1-13.

[5] G. Liu, "A method for constructing a model form for the Boltzman equation", Phys. Fluids, 2:2 (1990), 277-280. 
[6] Y. Zheng, H. Struchtrup, "Ellipsoidal statistical Bhatnagar-Gross-Krook model with velocity-dependent collision frequency", Phys. Fluids, 17:2 (2005), 127103, 17 pp.

[7] А. А. Латышев, А. А. Юшканов, "Тепловое и изотермическое скольжение в новом модельном кинетическом уравнении Лиу", Писъма в ЖТФ, 23:14 (1997), 13-16.

[8] А.А. Латышев, А.А. Юшканов, "Скачок температуры и слабое испарение в молекулярных газах", ЖЭТФ, 114:3(9) (1998), 956-971.

[9] М.Н. Коган, Динамика разреженного газа, Наука, М., 1967.

[10] К. Черчиньяни, Теория и приложения уравнения Болъцмана, Мир, М., 1978.

[11] А. А. Латышев, А. А. Юшканов, "Аналитическое решение граничной задачи для уравнения Шахова с частотой столкновений, пропорциональной скорости молекул”, Изв. РАН. Сер. МЖГ, 2003, № 4, 144-157.

[12] Н.Б. Енгибарян, А.Х. Хачатрян, "Вопросы нелинейной теории динамики разреженного газа", Матем. моделирование, 16:1 (2004), 67-74.

[13] В. А. Амбарцумян, "О некоторых нелинейных задачах теории переноса излучения", Теория звездных спектров, ред. В.В.Соболев, В. Г. Горбацкий, В.В.Иванов, Наука, M., 91-104.

[14] Н. Б. Енгибарян, "Об одной задаче нелинейного переноса излучения”, Астрофизика, 26:1 (1966), 31-36.

[15] А.Х. Хачатрян, “Об аналитическо-численном решении задачи Куэтта в рамках БГК модели уравнения Больцмана в нелинейном и линейном случаях", Вестн. РоссийскоАрмянского (Славянского) университета, 2 (2014), 16-32.

[16] О.А. Коленчиц, Тепловая аккомодачия систем газ - твердое тело, Изд-во "Наука и техника", Минск, 1977.

Поступила в редакцию 14.06.2016, после доработки 29.07.2016 